\title{
In Vitro Comparison of Aerosol Delivery Using Different Face Masks and Flow Rates With a High-Flow Humidity System
}

\author{
Hui-Ling Lin MSc RRT RN FAARC, Robert J Harwood MSA RRT FAARC, \\ James B Fink PhD RRT FAARC, Lynda T Goodfellow EdD RRT AE-C FAARC, \\ and Arzu Ari PhD RRT PT CPFT FAARC
}

\begin{abstract}
BACKGROUND: Aerosol drug delivery to infants and small children is influenced by many factors, such as types of interface, gas flows, and the designs of face masks. The purpose of this in vitro study was to evaluate aerosol delivery during administration of gas flows across the range used clinically with high-flow humidity systems using 2 aerosol masks. METHODS: A spontaneous lung model was used to simulate an infant/young toddler up to $2 \mathrm{y}$ of age and pediatric breathing patterns. Nebulized salbutamol by a vibrating mesh nebulizer positioned at the inlet of a high-flow humidification system at gas flows of 3,6 , and $12 \mathrm{~L} / \mathrm{min}$ was delivered via pediatric face masks to a pediatric face mannequin attached to a filter. Aerosol particle size distribution exiting the vibrating mesh nebulizer and at the mask position distal to the heated humidifier with 3 flows was measured with a cascade impactor. Eluted drug from the filters and the impactor was analyzed with a spectrophotometer $(n=3)$. Statistical analysis was performed by analysis of variance with a significant level of $P<.05$. RESULTS: The inhaled mass was between $2.8 \%$ and $8.1 \%$ among all settings and was significantly lower at $12 \mathrm{~L} / \mathrm{min}(P=.004)$ in the pediatric model. Drug delivery with pediatric breathing was greater than with infant breathing $(P=.004)$. The particle size distribution of aerosol emitted from the nebulizer was larger than the heated humidified aerosol exiting the tubing $(P=.002)$, with no difference between the 3 flows $(P=.10)$. CONCLUSIONS: The flows of gas entering the mask and breathing patterns influence aerosol delivery, independent of the face mask used. Aerosol delivery through a high-flow humidification system via mask could be effective with both infant and pediatric breathing patterns. Key words: pediatric; aerosol delivery; high-flow humidification; aerosol mask; gas flows; particle size distribution; vibrating mesh nebulizer. [Respir Care 2015;60(9):1215-1219. (C) 2015 Daedalus Enterprises]
\end{abstract}

\section{Introduction}

Aerosol therapy along with oxygen therapy is often administered to hospitalized infants and children. Chua et $\mathrm{al}^{1}$

\footnotetext{
Ms Lin is affiliated with the Respiratory Therapy Program, College of Medicine, Chang Gung University, Taoyuan, Taiwan, Republic of China. Mr Harwood and Drs Fink, Goodfellow, and Ari are affiliated with the Division of Respiratory Therapy, Georgia State University, Atlanta, Georgia. Dr Fink is also affiliated with James B Fink LLC, San Mateo, California.
}

This joint research project was developed under a Memorandum of Cooperation between Chang Gung University and Georgia State University to promote international cooperation in education and research.

Ms Lin presented a version of this paper in an open forum section of the 58th International Respiratory Congress, held November 10-13, 2012, in New Orleans, Louisiana. reported lung deposition of $0.3-1.6 \%$ in sleeping infants from 0.3 to $1.4 \mathrm{y}$ of age using a jet nebulizer operating at $9 \mathrm{~L} / \mathrm{min}$. However, administering aerosol therapy to infants and children is a challenge, as the device noise, mask fit, and cold air can irritate these small patients, causing

\footnotetext{
Dr Fink is a consultant to Aerogen, Ansun, Bayer, Boerhinger Ingleheim, Dance Biopharm, Novartis, Ony, Parion, Aridis, and the World Health Organization. The other authors have disclosed no conflicts of interest.

Correspondence: Hui-Ling Lin MSc RRT RN FAARC, Respiratory Therapy Program, College of Medicine, Chang Gung University, 259 WenHaw 1st, Kwei-Shan, Taoyuan, Taiwan, Republic of China. E-mail: huilingrrt@gmail.com
}

DOI: $10.4187 /$ respcare. 03595 
various degrees of distress associated with decreased aerosol delivery. ${ }^{2}$

\section{See the Related Editorial on Page 1365}

High-flow, heated humidified oxygen therapy provides oxygen gas flows higher than the patient's inspiratory flow, and also provides adequately warmed and humidified gas to conducting airways to reduce airway damage associated with gas conditioning. Aerosol therapy through the highflow nasal cannula has been suggested as a reasonable interface option for aerosol administration, with an inhaled dose comparable to standard aerosol therapy that is well tolerated by the child. ${ }^{3,4}$ In vitro studies have shown the efficiency of delivering bronchodilators via high-flow nasal cannula, but the inhaled dose decreased significantly as the flow increased. ${ }^{3-5}$ Although clinicians have adopted such technology, the question arises: how much aerosol would be inhaled using a similar setup with an aerosol face mask? Studies have shown that the design of the face mask affects the amount of aerosolized drug delivery. ${ }^{6-8}$ The deposition of aerosolized drug delivered with a face mask through a high-flow heated humidified oxygen system has not been examined.

The purpose of this in vitro study was to compare the efficiency of aerosol delivery with 2 different face mask designs using a high-flow humidity system and variable gas flows. We hypothesize that the inhaled dose will be inversely related to flow through the system, with differences between infant and pediatric breathing patterns.

\section{Methods}

This study was conducted in the Respiratory Research Laboratory in the Respiratory Therapy Department at Chang Gung University (Taoyuan, Taiwan, Republic of China).

\section{Lung Model}

A lung simulator (ASL 5000, IngMar Medical, Pittsburgh, Pennsylvania) was set to represent spontaneous breathing patterns for an infant/young toddler up to $2 \mathrm{y}$ of age (weight range $10-15 \mathrm{~kg}$ ) and for a child (weight range $25-30 \mathrm{~kg}$ ) . Infant/young toddler breathing pattern parameters were set at tidal volume $=100 \mathrm{~mL}$, inspiratory time $=0.7 \mathrm{~s}$, and breathing frequency $=30$ breaths $/ \mathrm{min}$, and pediatric parameters were tidal volume $=250 \mathrm{~mL}$, inspiratory time $=1.0 \mathrm{~s}$, and breathing frequency $=$ 20 breaths/min. To represent drug deposition distal to the upper airway, a face and anatomical upper airway of a child cardiopulmonary resuscitation mannequin was attached distal to the hypo-pharynx to a bacterial filter

\section{QUICK LOOK}

\section{Current knowledge}

Aerosol drug delivery to infants and small children can be influenced by a number of factors including the type of interface, gas flow, and the face mask design. The presence of humidity can also alter aerosol delivery. Previous research suggests that humidity can both facilitate and interfere with aerosol deposition.

\section{What this paper contributes to our knowledge}

Aerosol delivery with high-flow humidification is affected by gas flow and breathing patterns, independent of the face mask used. Gas flows $>12 \mathrm{~L} / \mathrm{min}$ decreased the inhaled drug delivered. During aerosol delivery through a high-flow humidification system via mask at flows $>12 \mathrm{~L} / \mathrm{min}$, medication dose may need to be increased to achieve the desired effect.

(GaleMed, Taipei, Taiwan, Republic of China) with a filtration rate $>99.999 \%$ at particle size of $0.3 \mu \mathrm{m}$, and with an internal dead space of $33 \mathrm{~mL}$. Figure 1 shows the configuration of the experimental design.

\section{Inhaled Dose Measurements}

A unit-dose of salbutamol $(5.0 \mathrm{mg} / 2.5 \mathrm{~mL}$; GlaxoSmithKline, Philadelphia, Pennsylvania) was placed in the reservoir of a vibrating mesh nebulizer (VMN; Aeroneb Solo, Aerogen, Mountain View, California) with continuous aerosol generation. The VMN was run until $30 \mathrm{~s}$ after no generated aerosol was visible. Each experiment was repeated in triplicate. Results were expressed as the proportion of the dose delivered distal to the airway.

Compressed air from a central piping system at $50 \mathrm{psi}$ was metered through a pressure compensated flow meter at 3,6, and $12 \mathrm{~L} / \mathrm{min}$, passing through a $22 \mathrm{~mm} \mathrm{~T}$-adapter holding the VMN and attached to the inspiratory inlet of the heated humidifier (MR410, Fisher \& Paykel, Auckland, New Zealand). The temperature control of the MR410 humidifier was a scale of $1-9$, and the maximum setting (9) was set in the present study. The delivered temperature with set flows was approximately $34-35^{\circ} \mathrm{C}$.

A heated wire circuit from the humidifier was attached to an OxyKid aerosol mask (SouthMedic, Barrie, Ontario, Canada) and the Dragon aerosol mask (Cardinal Health, Dublin, Ohio). Figure 2 demonstrates the configuration of the 2 masks. Both aerosol masks offer child-friendly elements to improve compliance, with substantial difference in design. The OxyKid is a front-loaded mask with gas directed to the face at a $90^{\circ}$ angle with 3 large holes on 


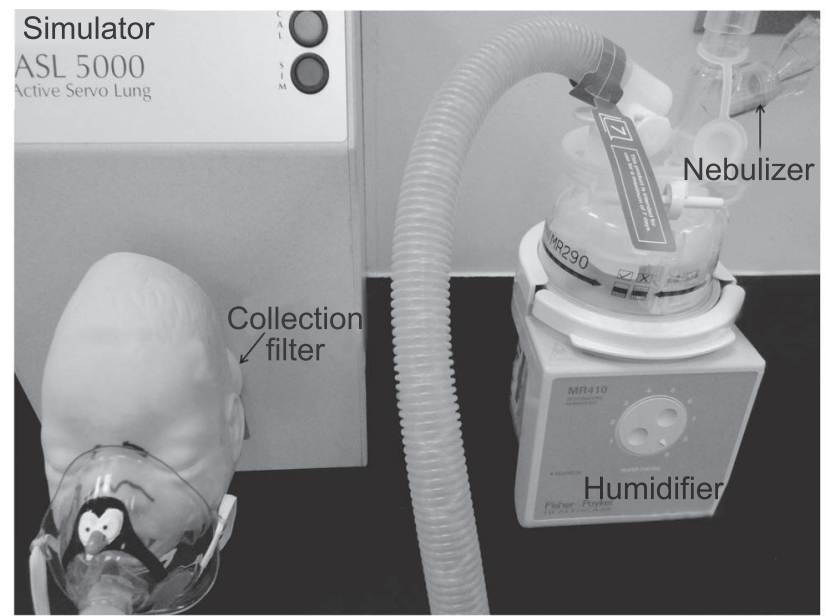

Fig. 1. Configuration of the experimental design.

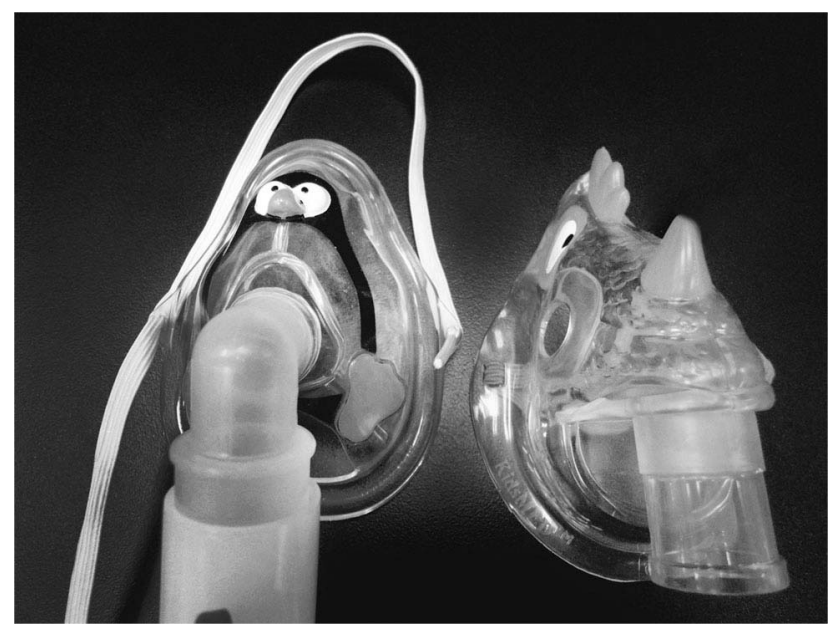

Fig. 2. The OxyKid mask (left) and the Dragon mask (right).

both sides and the bottom. In contrast, the Dragon mask is a bottom-loaded design that directs the aerosol and gas flow up to the face from below the nose, with smaller $1 \mathrm{~cm}$ side-holes that somewhat restrict gas entering or leaving the sides of the mask. The same size mask was used with both sets of breathing parameters.

\section{Particle Size Measurement}

Aerosol generated from the VMN was characterized using an Anderson cascade impactor (Thermo Fisher Scientific, Waltham, Massachusetts) to measure aerosol particle size distribution in accordance with United States pharmaceutical standards, at a flow of $28.3 \mathrm{~L} / \mathrm{min}$. Aerosol was sampled as it was emitted from the VMN at ambient condition, and after passing through the humidifier and the heated tubing at the position of the mask with flows of 3 , 6 and $12 \mathrm{~L} / \mathrm{min}$. All measurements were repeated in triplicate.

\section{Drug Measurement}

Drug collected on the filter and impactor stages was eluted with distilled water for $1 \mathrm{~min}$ with gentle agitation and analyzed with a spectrophotometer (Thermo Fisher Scientific) with wavelength at $276 \mathrm{~nm}$. The spectrophotometer was calibrated and set to zero before each trial. The concentration of the sample solution and the amount of drug were calculated from a known concentration/absorbency regression $\left(r^{2}>.99\right)$.

\section{Data Analysis}

The amount of drug eluted from the filter was quantified in $\mu \mathrm{g} / \mathrm{mL}$ and expressed as a percentage of the total dose placed in the VMN. The median mass aerodynamic diameter (MMAD) and the geometric standard deviation (GSD) were calculated from the amount of drug deposited on the impactor plates and the throat. The normality of data distribution was analyzed. The mean \pm SD was calculated for each component of the drug mass eluted from the inspiration filter. Statistical analyses were performed using SPSS 18.0 (SPSS, Chicago, Illinois). Independent $t$ test and one-way analysis of variance with Bonferroni test were used for statistical analysis, and $P<.05$ was used for statistical significance. To test our hypothesis that the inhaled dose was inversely related to flow through the system, Pearson correlation analysis was used.

\section{Results}

\section{Inhaled Drug}

Inhaled drug delivered expressed as percentage (mean \pm SD) of total dose in the infant/young toddler model is shown in Table 1. The inhaled mass in the OxyKid mask was between $2.8 \%$ and $7.3 \%$, and it was significantly lower at $12 \mathrm{~L} / \mathrm{min}$ than at 3 and $6 \mathrm{~L} / \mathrm{min}(P=.004)$. Drug deposition with the Dragon mask was $3.2-8.1 \%$ with a significant difference $(P=.003)$ in the pediatric model. The inhaled mass with the Dragon mask under the infant/young toddler breathing pattern was significantly greater at $6 \mathrm{~L} / \mathrm{min}$ than at $12 \mathrm{~L} / \mathrm{min}(P<.001)$. Drug delivery with the pediatric breathing pattern was greater than infant/young toddler pattern with a mean $\pm \mathrm{SD}$ of $6.4 \pm 1.8 \%$ versus $4.6 \pm 1.5 \%$, respectively $(P=.004)$. Inhaled dose was similar with OxyKid and Dragon masks (mean \pm SD of $5.0 \pm 1.8 \%$ and $6.0 \pm 1.9 \%$, respectively, $P=.12$ ).

The correlation between the inhaled mass and flow showed $\mathrm{r}^{2}=.39(P<.001)$. The regression equation showed a negative relationship between inhaled mass and flow $(\mathrm{Y}=7.786-0.328 \times$ flow $)$. 
Table 1. Inhaled Mass, With Both Infant and Pediatric Breathing Patterns, at Flows of 3, 6, and $12 \mathrm{~L} / \mathrm{min}$

\begin{tabular}{lccccr}
\hline \hline \multirow{2}{*}{ Model } & \multicolumn{3}{c}{ Mask and Flow } \\
\cline { 2 - 4 } & \multicolumn{3}{c}{ OxyKid } & & \multicolumn{2}{c}{ Dragon } \\
\cline { 2 - 5 } & $3 \mathrm{~L} / \mathrm{min}$ & $6 \mathrm{~L} / \mathrm{min}$ & $12 \mathrm{~L} / \mathrm{min}^{*}$ & $3 \mathrm{~L} / \mathrm{min}$ & $6.2 \pm 1.38$ \\
\hline Infant & $6.4 \pm 1.2$ & $4.2 \pm 0.74$ & $2.8 \pm 0.16$ & $4.7 \pm 0.82$ & $3.2 \pm 0.44$ \\
Pediatric & $7.3 \pm 0.9$ & $5.8 \pm 0.62$ & $3.4 \pm 0.36$ & $8.0 \pm 1.09$ & $8.1 \pm 1.12$
\end{tabular}

Inhaled mass was expressed as percentage (mean $\pm \mathrm{SD}$ ) of total dose inhaled with the 2 masks.

* Drug delivery with $12 \mathrm{~L} / \mathrm{min}$ was less than other 2 flows $(P<.05)$.

Table 2. Aerosol Particle Size Distributions Expressed as MMAD and GSD of Nebulizer Output and Mask Output at 3, 6, and 12 L/min

\begin{tabular}{lcccc}
\hline \hline & \multicolumn{3}{c}{ Distributions } & \\
\cline { 2 - 5 } & Nebulizer & $3 \mathrm{~L} / \mathrm{min}$ & $6 \mathrm{~L} / \mathrm{min}$ & $12 \mathrm{~L} / \mathrm{min}$ \\
\hline $\operatorname{MMAD}(\mu \mathrm{m})$ & $4.0 \pm 0.14^{*}$ & $2.8 \pm 0.12$ & $3.3 \pm 0.15$ & $2.8 \pm 0.36$ \\
$\operatorname{GSD}(\mu \mathrm{m})$ & $2.1 \pm 0.06$ & $1.7 \pm 0.2$ & $1.9 \pm 0.25$ & $1.7 \pm 0.15$
\end{tabular}

Aerosol particle size distributions are expressed as MMAD and GSD.

* Greater than output from mask at all 3 flows $(P<.002)$.

$\mathrm{MMAD}=$ median mass aerodynamic diameter

$\mathrm{GSD}=$ geometric SD

\section{Aerosol Particle Size Distributions}

Aerosol characterizations with the MMAD and GSD are presented in Table 2 for measurements at the exit of the VMN at ambient conditions, and with the 3 flows after passing through a heated humidifier and delivery tubing. The MMAD of aerosol emitted from the VMN was larger than the heated humidified aerosol exiting the tubing at 3 , 6 , and $12 \mathrm{~L} / \min (P=.002)$ with no difference between the 3 flows $(P=.10)$.

\section{Discussion}

Our results confirmed that variation of flow to a mask influences aerosol drug deposition. In general, the higher the flow delivered, the less aerosol delivered to the patient, although we did see an exception with increased or similar drug delivery at $6 \mathrm{~L} / \mathrm{min}$ as opposed to $3 \mathrm{~L} / \mathrm{min}$ with the Dragon mask with infant/young toddler and pediatric breathing patterns, respectively. Drug delivery through a mask is also influenced by breathing pattern; the lower the tidal volume and minute ventilation, the less aerosol delivered to the patient. In contrast, delivered MMAD was not affected by changes in delivered flow.

Comparing our model to a similar lung model by Ari et $\mathrm{al}^{3}$ on the influence of flow through the high-flow nasal cannula, as the total flow increases, the inhaled dose decreases. The inhaled dose was approximately 5 -fold higher with an oxygen flow of $3 \mathrm{~L} / \mathrm{min}$ than with $6 \mathrm{~L} / \mathrm{min}$. A major difference between delivery via nasal prongs and the aerosol mask may be the size of reservoir represented by the nasopharynx and the volume of the mask. Our results showed that the inhaled dose was decreased significantly only at $12 \mathrm{~L} / \mathrm{min}$. With an infant/young toddler breathing pattern, the drug delivery was $10.6 \%$ with nasal cannula and $6.5 \%$ with mask at $3 \mathrm{~L} / \mathrm{min}$. This suggests that aerosol delivery can be more efficient with a nasal cannula at lower flows. However, as the flow increases, a mask interface may be more efficient for aerosol delivery.

In children, aerosol delivery with a jet nebulizer is influenced by the design of the aerosol mask.6,7,9,10 Harris and Smaldone ${ }^{10}$ demonstrated that, as an accessory for a jet nebulizer, the front-loaded mask configuration was more efficient than the bottom-loaded mask configuration, which is in contrast to our study. Our data showed that the designs of the face masks we tested do not influence aerosol delivery with a VMN at the flows tested. A jet nebulizer requires a gas source of $50 \mathrm{psig}$ at $6-10 \mathrm{~L} / \mathrm{min}$. As the aerosol generated by a jet nebulizer travels to the attached device, the first impaction may influence drug deposition.

A study by Smaldone et $\mathrm{al}^{11}$ has suggested increased particle inertia along the edge of the mask influences drug deposition. They suggested a difference between front and bottom loading mask designs. However, with a VMN, the exit velocity of the aerosol is low $(<4 \mathrm{~m} / \mathrm{s})$. In our study, the VMN was placed upstream of the heated humidifier system. With the humidifier as a reservoir and first-line baffle, the effect of particle inertia was less evident; thus, 
the drug deposition was similar with 2 different aerosol masks.

Our results of reduced particle size distribution between nebulizer output and aerosol leaving the heated tubing are consistent with reports from Bhashyam et $\mathrm{al}^{4}$ using $3 \mathrm{~L} / \mathrm{min}$. However, we did not see a further reduction in particle size with the higher flows tested ( 6 and $12 \mathrm{~L} / \mathrm{min}$ ), suggesting that particle size is reduced but not flowdependent in the range tested.

\section{Limitations}

This was an in vitro model that simulated the breathing pattern of infant/young toddlers up to $2 \mathrm{y}$ of age and small children. Pediatric patients range from preterm infants to youth $18 \mathrm{y}$ of age with different ranges of tidal volume and breathing patterns. Inhaled mass may vary with these parameters. Additionally, the aerosolized drug was captured and measured at the position of the mouth with no simulation of upper airway deposition. Clinical trials are needed to verify and correlate in vitro differences to the physiological and clinical effects of aerosol delivery through highflow humidification system in patients.

\section{Conclusions}

Aerosol delivery with high-flow humidification is affected by the flows of gas entering a mask and the breathing patterns, independent of the face mask used. As gas flow increased to $12 \mathrm{~L} / \mathrm{min}$, the inhaled drug delivered decreased. Based on this in vitro study of aerosol delivery through a high-flow humidification system via mask, clinicians may want to consider increasing doses when administering higher flows.

\section{ACKNOWLEDGMENTS}

We thank Robert Trusty of Children Hospital and Clinics of Minnesota for providing research material.

\section{REFERENCES}

1. Chua HL, Collis GG, Newbury AM, Chan K, Bower GD, Sly PD, Le Souef PN. The influence of age on aerosol deposition in children with cystic fibrosis. Eur Respir J 1994;7(12):2185-2191.

2. Iles R, Lister P, Edmunds AT. Crying significantly reduces absorption of aerosolised drug in infants. Arch Dis Child 1999;81(2): 163-165.

3. Ari A, Harwood R, Sheard M, Dailey P, Fink JB. In vitro comparison of heliox and oxygen in aerosol delivery using pediatric high flow nasal cannula. Pediatr Pulmonol 2011;46(8):795-801.

4. Bhashyam AR, Wolf MT, Marcinkowski AL, Saville A, Thomas K, Carcillo JA, Corcoran TE. Aerosol delivery through nasal cannulas: an in vitro study. J Aerosol Med Pulm Drug Deliv 2008;21(2):181188.

5. Perry SA, Kesser KC, Geller DE, Selhorst DM, Rendle JK, Hertzog $\mathrm{JH}$. Influences of cannula size and flow rate on aerosol drug delivery through the Vapotherm humidified high-flow nasal cannula system. Pediatr Crit Care Med 2013;14(5):e250-256.

6. Lin HL, Restrepo RD, Gardenhire DS, Rau JL. Effect of face mask design on inhaled mass of nebulized albuterol, using a pediatric breathing model. Respir Care 2007;52(8):1021-1026.

7. Sangwan S, Condos R, Smaldone GC. Lung deposition and respirable mass during wet nebulization. J Aerosol Med Pulm Drug Deliv 2003;16(4):379-386.

8. Amirav I, Mansour Y, Mandelberg A, Bar-Ilan I, Newhouse MT. Redesigned face mask improves "real life" aerosol delivery for Nebuchamber. Pediatr Pulmonol 2004;37(2):172-177.

9. Lin HL, Wan GH, Chen YH, Fink JB, Liu WQ, Liu KY. Influence of nebulizer type with different pediatric aerosol masks on drug deposition in a model of a spontaneously breathing small child. Respir Care 2012;57(11):1894-1900.

10. Harris KW, Smaldone GC. Facial and ocular deposition of nebulized budesonide: effects of face mask design. Chest 2008;133(2):482488.

11. Smaldone GC, Sangwan S, Shah A. Facemask design, facial deposition, and delivered dose of nebulized aerosols. J Aerosol Med Pulm Drug Deliv 2007;20(Suppl 1):S66-S75.

This article is approved for Continuing Respiratory Care Education credit. For information and to obtain your CRCE

(free to AARC members) visit www.rcjournal.com

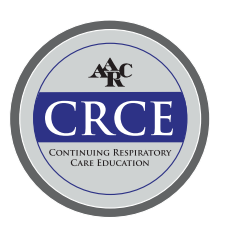

